East African Medical Journal Vol. 86 No. 10 October 2009

ACUTE CORONARY SYNDROMES AMONGST TYPE 2 DIABETICS WITH ISCHAEMIC ELECTROCARDIOGRAMS PRESENTING TO ACCIDENT AND EMERGENCY DEPARTMENT OF A KENYAN TERTIARY INSTITUTION

H. K. Nguchu, MBChB, MMed, Department of Medicine, Kenyatta National Hospital, P.O. Box 20723-00202, Nairobi, Kenya, M. D. Joshi, MBChB, MMed, Senior Lecturer, Department of Clinical Medicine and Therapeutics, and Clinical Epidemiology Unit and C.F.Otieno, MBChB, MMed, Senior Lecturer, Department of Clinical Medicine and Therapeutics, School of Medicine, College of Health Sciences, University of Nairobi, P. O. Box 19676-00202, Nairobi, Kenya

Request for reprints to: Dr. H. K. Nguchu, Department of Medicine, Kenyatta National Hospital, P.O. Box 20723-00202, Nairobi, Kenya

\title{
ACUTE CORONARY SYNDROMES AMONGST TYPE 2 DIABETICS WITH ISCHAEMIC ELECTROCARDIOGRAMS PRESENTING TO ACCIDENT AND EMERGENCY DEPARTMENT OF A KENYAN TERTIARY INSTITUTION
}

\author{
H. K. NGUCHU, M.D. JOSHI and C. F. OTIENO
}

\begin{abstract}
Objective: To determine the prevalence of acute coronary syndromes among type 2 diabetic patients presenting to Accident and Emergency department.

Design: Prospective cross-sectional study.

Setting: Kenyatta National Hospital, a tertiary teaching and referral hospital.

Subjects: Type 2 diabetic patients with ischaemic electrocardiograms (ECG).

Main outcome measures: Demographics, clinical symptoms, cardiovascular status and risk factors - central obesity, hypertension, dyslipidaemia, smoking.

Results: From 12,307 accident and emergency attendees, 400 ( 33\%) diabetics aged $\geq 30$ years were screened with a resting ECG and $95(24 \%)$ with ischaemic ECG were recruited; age range $41-87$ years, $60 \%$ were male; diabetes duration ranged $0-30$ years with $8.4 \%$ being newly diagnosed. The commonest enrolling ECG feature was nonspecific ST-T changes. The commonest presenting complaint were fatigue and dyspnoea. Majority had three coronary artery disease (CAD) risk factors: obesity $86 \%$, elevated LDL $73 \%$ and hypertension $60 \%$. Therapy in use was OHA $43 \%$, insulin $42 \%$, insulin and $\mathrm{OHA} 1 \%$; prophylactic aspirin $14.7 \%$ and statins $8.4 \%$. Thirty four $(35.8 \%)$ were classified as acute coronary syndrome (ACS); 29 (30.5\%) acute myocardial infarction (ACS-AMI) and five (5.2\%) unstable angina (ACS-UA). Majority (79.4\%) of the ACS presented more than six hours after symptom onset and majority had features of acute left ventricular failure.

Conclusions: Acute coronary syndrome accounted for $35 \%$ of the morbidity in type 2 diabetics with ischaemic ECG's presenting to $\mathrm{KNH}$ accident and emergency department; patients presented late and $80 \%$ were not on CAD prophylactic therapy.
\end{abstract}

\section{INTRODUCTION}

The global number of individuals with diabetes is projected to increase markedly from 171 million (2.8\% of the world's population) in 2000 to 366 million $(6.5 \%)$ in 2030; 298 million of whom, will live in developing countries (1). Cardiovascular disease, with its attendant morbidity and mortality, particularly in the diabetic population, is on the rise in the developing countries (2) with mortality expected to increase from an estimated 9 million in 1990 to 19 million by 2020 (3).

Coronary artery disease (CAD) is a major cause of morbidity and mortality amongst patients with diabetes mellitus. Several studies have shown that compared to non-diabetics, diabetics have a higher prevalence of $\mathrm{CAD}$, a greater extent of coronary ischaemia, and are more likely to have acute myocardial infarction (AMI), and silent myocardial ischaemia $(4-6)$.

Coronary artery disease is a growing epidemic on the African continent, on account of the increasing prevalence of the classical risk factors for $\mathrm{CAD}$, with a regularly reported concurrence of multiple risk factors in the sameindividual (7). In the INTERHEART Africa study (8), history of smoking, diabetes, hypertension, abdominal obesity, and ratio of apolipoprotein B to apolipoprotein A-1 provided for a population attributable risk of $89.2 \%$ for AMI. Non-specific ST$\mathrm{T}$ wave ECG changes has also been shown to have CAD prediction power and can be considered as a risk factor (9).

In observational studies of CAD in diabetes, incidence rates of AMI have varied widely mainly as 
a function of ethnic background, inclusion criteria, definition of end points, and duration of follow-up (10-12). There is limited local data on the prevalence and presenting characteristics of ACS amongst the diabetics in our population.

Consequently, the aim of this study was to determine the prevalence and presenting characteristics of acute coronary syndromes amongst type 2 diabetics with ischaemic ECG's.

\section{MATERIALS AND METHODS}

During the period of July 2007 to October 2007 all type 2 diabetic patients, with a prior chart diagnosis/treatment or A\&E newly diagnosed and aged $>30$ years, presenting to the Kenyatta National Hospital and Emergency Department (KNH A\&E) with medical complaints were screened with a 12 lead resting ECG using a standardised portable machine. Those with ECG features of ischaemia were consecutively recruited and underwent a thorough clinical evaluation including weight, waist and hip circumferences and blood drawn for measurement of serial serum Troponin I and fasting lipid levels.

Ischaemia ECG features were defined as follows: Abnormal $Q$ waves: any $Q$ waves in leads V1 to V3 or $\mathrm{Q}$ wave $\geq 30 \mathrm{~m}$ sec in leads I, II, aVL, aVF, or V4 - V6; present in any two contiguous leads and $\geq 1$ $\mathrm{mm}$ in depth. ST elevation: $1 \mathrm{~mm}$ from the baseline measured at 0.08 seconds from the J point, in at least two contiguous precordial leads or in at least two adjacent limb leads. ST depression not associated with ventricular hypertrophy: horizontal, down sloping or up sloping depression at $>1 \mathrm{~mm}$ from the baseline measured at 0.08 seconds from the J point. $\mathrm{T}$ wave inversion not associated with ventricular hypertrophy and Left Bundle Branch Block by standard conventional criteria (13).

Patients were classified as hypertensive on basis of a chart diagnosis or treatment for hypertension. Newly diagnosed patients were diagnosed on the basis of JNC 7 criteria by the attending A\&E physician. Cigarette smoking status was classified as either current smoker: last smoked within the past one year or non - smoker: last smoked more than a year ago or had never smoked. A positive family history of CAD was defined as CAD in a first degree relative occurring before age of 60 years (14). Waist circumference was measured in $\mathrm{cm}$, in the horizontal plane, at the level of the narrowest part of the torso between the lowest rib and the top of the pelvis, as seen from the anterior view, at the end of a normal expiration (15). A measured value of $\geq$ $102 \mathrm{~cm}$ in males and $\geq 88 \mathrm{~cm}$ in females was classified as Central obesity as defined by NCEP / ATPIII (16). Thehip circumference was measured in centimeters, as the maximum circumference at the level of the greater trochanters in the horizontal plane (17). The Waist Hip Ratio was calculated as the ratio of the former to the latter and defined by WHO criteria as abnormal if the ratio was $>0.90$ in males and $>0.85$ in females (18).

Two mililiters of blood was drawn for measurement of serum Troponin I and if the initial level was normal, a second sample was drawn 8 hours later. The samples were assayed by a dedicated study technician at the KNH laboratory with the Minividas Troponin I kit by HASS Ltd. Serum Troponin I levels $>0.01 \mathrm{ng} / \mathrm{ml}$ were considered elevated and therefore diagnostic of an acute myocardial infarction, representing the 99th percentile value for the kitutilised (13). Two mls of blood was drawn after an 8 hour fast for fasting lipid levels. The samples were assayed by a dedicated study technician at the Department of Internal Medicine laboratory, University of Nairobi with the Human kits by HUMAN Ltd. According to the NCEP / ATPIII guidelines (16), the following levels were considered abnormal:HDL-C $<1.03 \mathrm{mmol} / \mathrm{I}$ (for both genders); Triglycerides $>1.7 \mathrm{mmolll}$ and LDL-C $>1.8 \mathrm{mmol} / 1$.

Study patients were classified as ACS or Non-ACS on basis of the following criteria; Acute myocardial infarction: elevated serum Troponin I; unstable angina: normal serum Troponin I with concurrent angina; Non-ACS: normal serum Troponin I without concurrent angina or angina equivalent dyspnoea.

The study was approved by the KNH ethics committee. Data was analysed with SPSS version 15.0 software. Continuous data was analysed into means and categorical data were analysed into percentages with the corresponding 95\% confidence intervals. Prevalence rates were calculated similarly. Statistical significance was defined as a two tailed p-value of less than or equal to 0.05 .

\section{RESULTS}

Over a four month period between 1st July 2007 to 31st October 2007,12,307 medical case patients were attended to at Kenyatta National Hospital Accident and Emergency Department (KNH A\&E), 400 (33\%) of whom were diabetics aged over 30 years. The latter group comprised our study target population and were consecutively screened with an index resting 12 lead ECG; 95 (24\%) were classified as ischaemic ECG and were recruited. Over the study recruitment period the numbers screened and proportion recruited on a weekly basis did not vary considerably; with a weekly average of 22 individuals screened and $22 \%$ recruited. (Figure 1). Age ranged from 41-87 years with a mean age of 63.3 years; $60 \%$ were male; $56 \%$ were either retired of unemployed; $58 \%$ had attained primary level of education whereas only $42 \%$ had attained secondary or above level of education. Minority of the patients were newly diagnosed diabetics (8.4\%); diabetic disease duration ranged from $0-30$ years with a mean of 11 years. 
Figure 1

Weekly screening and recruitment of diabetic patients

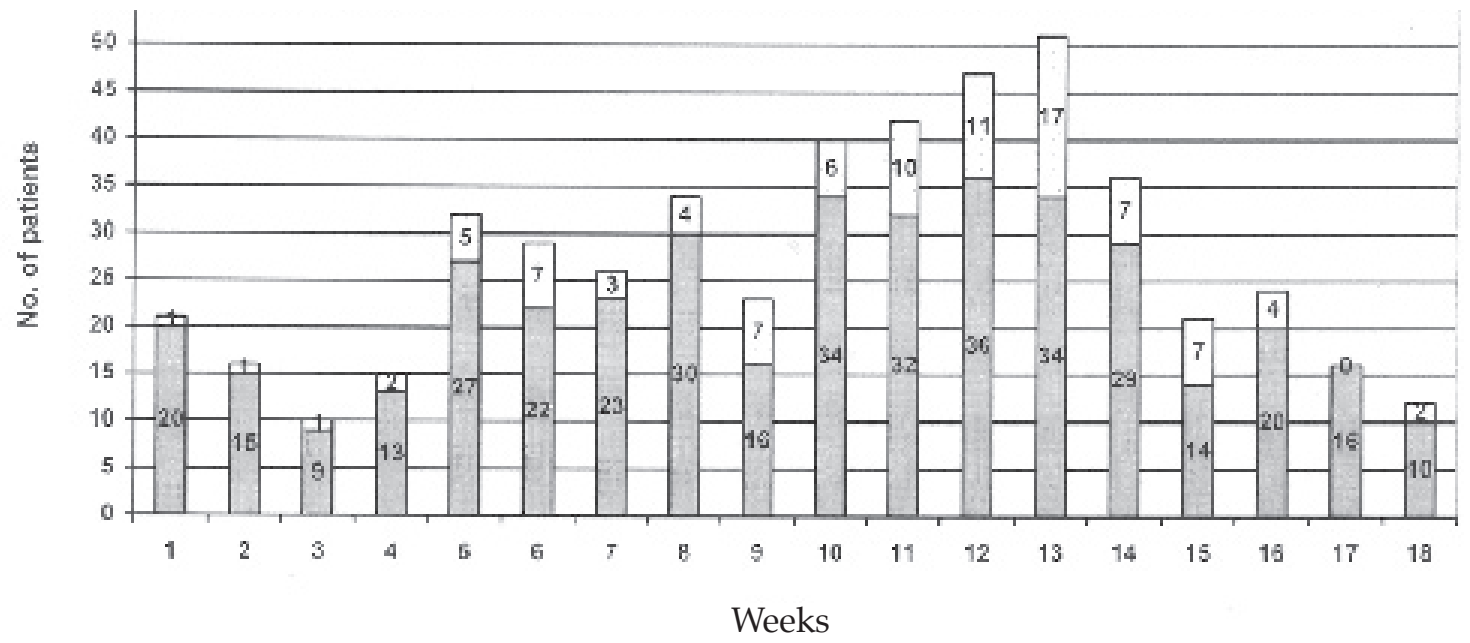

Screened patient $\square$ Recruited patients

Enrollment qualifying EGG included $41 \% \mathrm{~T}$ wave inversion, $18 \%$ ST depression and 15\% with LBBB. Ten patients hadST elevation and mixed features each and five patients demonstrated abnormal $\mathrm{Q}$ waves. Nine patients were in atrial fibrillation and the rest in normal sinus rhythm. (Table1)

Table 1

ECG characteristics of the study population

\begin{tabular}{lll}
\hline ECG feature & No. & $(\%)$ \\
\hline Defining Criteria - Ischaemic ECG features & & \\
$\quad$ T wave inversion in absence of LVH & 39 & 41.1 \\
ST depression & 17 & 17.9 \\
Left Bundle Branch Block & 14 & 14.7 \\
ST elevation & 10 & 10.5 \\
Multiple ischaemic features & 10 & 10.5 \\
Abnormal Q waves & 5 & 5.3 \\
Cardiac rhythm & & \\
$\quad$ Sinus rhythm & 86 & 90.5 \\
Atrial fibrillation & 9 & 9.5 \\
\hline
\end{tabular}

The commonest A\&E presenting complaint was exertional dyspnoea in $51.6 \% ; 30.5 \%$ were in NYHA Class III/IV; rest angina $16.8 \%$, epigastric pain $12.6 \%$ and cerebrovascular accident $4.2 \%$ (Figure 2). All patients had at least one other cardiovascular risk factor, with the majority having more than three factors; Obesity by WHR 86\%, elevated LDL 73\% obesity by waist circumference (WC) $62 \%$ and hypertension $60 \%$. Cigarette smoking wasinfrequent at $8.4 \%$ as was prior MI and family history both at $1 \%$. ( Figure 3). Median and range lipid values were total cholesterol 4.5 (1.4-10.5) mmol/I, LDL-C 2.4
(0.5-9.4) $\mathrm{mmol} / \mathrm{l}$, HDL 0.9 ( 0.1-2.4) $\mathrm{mmol} / \mathrm{I}$ and triglycerides $1.1(0.2-7.7) \mathrm{mmol} / 1$. Dyslipidaemia was documented in 95\%; 73\% elevated LDL-C, $54.7 \%$ depressed HDL and 30\% elevated triglycerides (TGs); $51.6 \%$ had only one lipid abnormality; $36.8 \%$ had two abnormalities while $6.3 \%$ had all three lipid abnormalities (Figure 5). Most women (97\%) were postmenopausal and all smokers were male. Women were more likely to be classified as obese on basis of the WC (male $43.9 \%$; female $89.5 \%$, p - 0.001); whereas on the basis of WHR obesity was equally distributed among the genders. 
Figure 2

Accident and emergency presenting symptoms of study patients

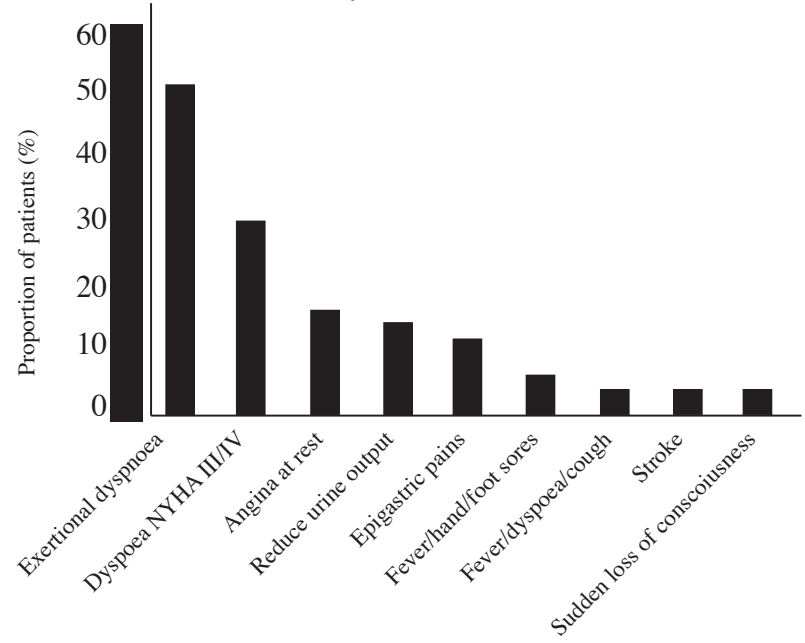

Figure 3

Cardiovascular risk factors in the study population $(n=95)$

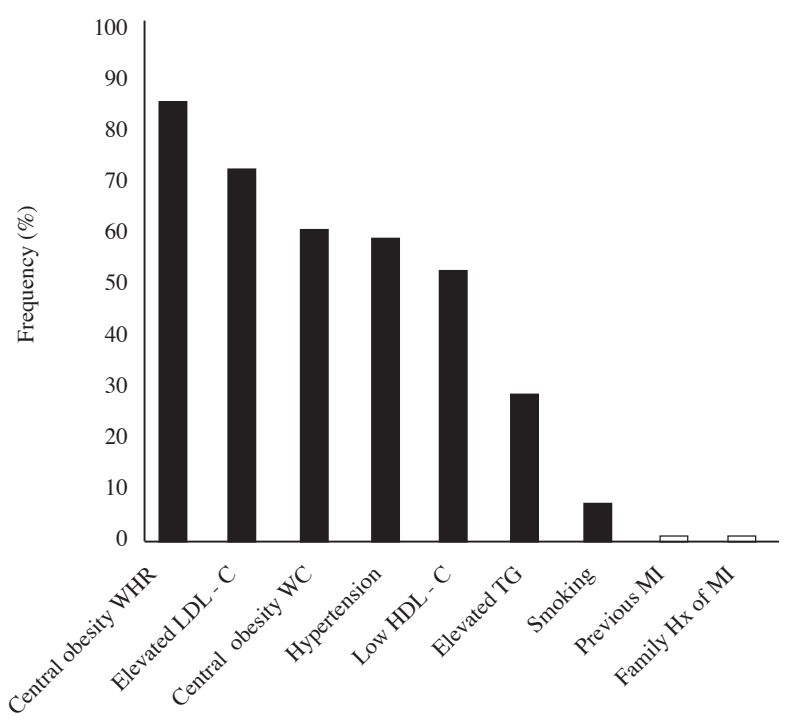

Figure 4

Flow chart of patients in study

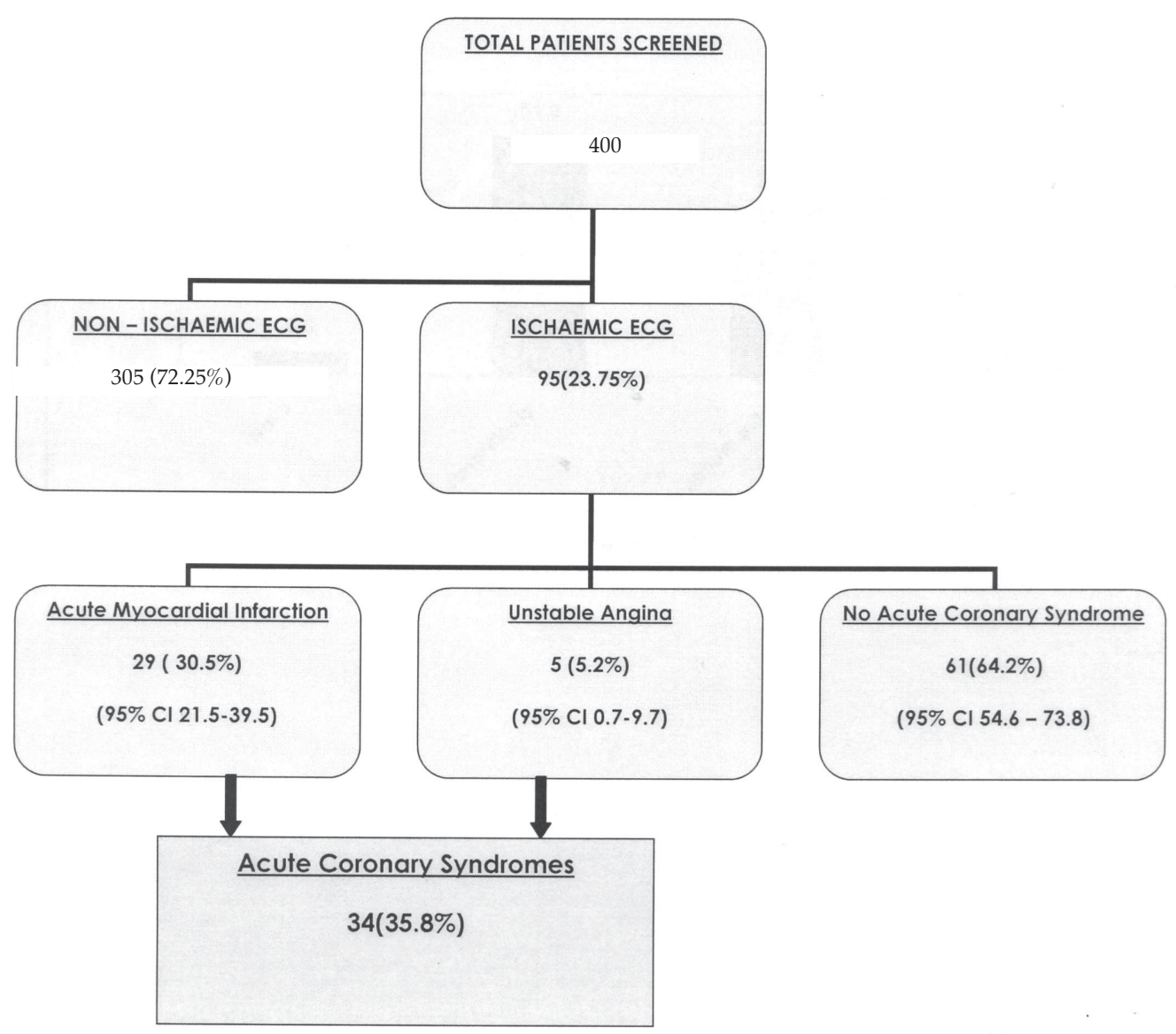


Figure 5

Occurrence of combinations of elevated LDLC/low HDLC and elevated triglycerides

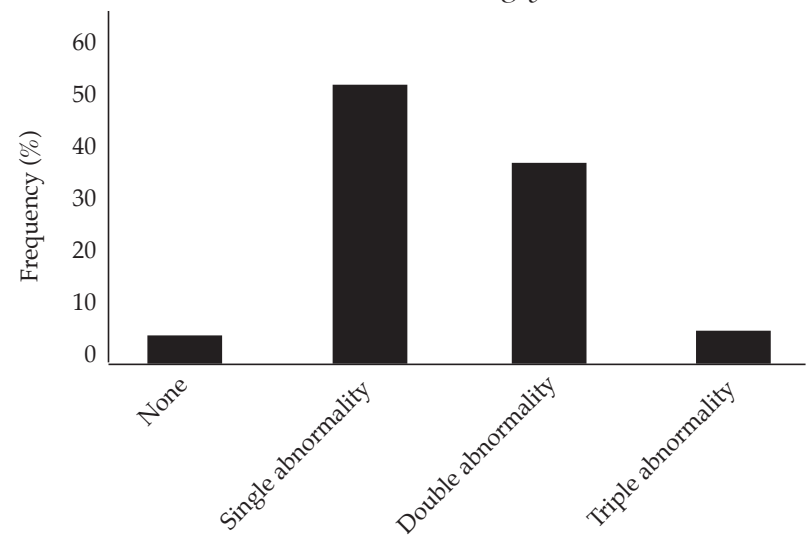

There was no statistically significant difference between the male and female patients with regard to the prevalence of hypertension $(p=0.4)$, central obesity as defined by waist-hip ratio $(p=0.6)$ and elevated LDL - Cholesterol ( $p=0.6$ ).

Anti-diabetic therapy utilised prior to A\&E presentation included oral hypoglycaemic agents (OHA) in 43\%, insulin 42\%, insulin and OHA $1 \%$ and dietary control in $5.3 \%$. Only $14.7 \%$ and $8 \mathrm{~A} \%$ of the patients in the study population were on aspirin and statins therapy respectively.

On the basis of clinical presentation and Troponin assays $34(35.8 \%)$ participants were classified as having an acute coronary syndrome (ACS); $29(30.5 \%)$ acute myocardial infarction (ACSAMI), five (5.2\%) unstable angina (ACS-UA) and 61 $(64.2 \%)$ as non-ACS presentations. (Figure 4).The most common symptoms amongst the patients with ACS were breathing difficulty and fatigue; 47\% presented with typical angina whereas $52.9 \%$ had atypical symptoms. Majority $(79.4 \%)$ of ACS patients presented to the A\&E six hours or more after onset of symptoms; the mean duration of symptoms being 5.5 days and majority had features of acute left ventricular failure (Figure 6).

\section{Figure 6}

Cardiovascular status of patients with acute myocordial infarction

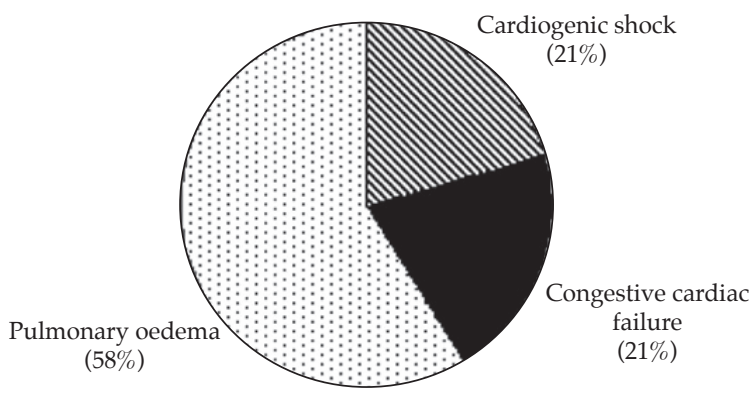

No statistically significant difference was evident between patients with and without acute coronary syndromes, in terms of age, gender, duration of diabetes or cardiovascular risk factors; the only exception being hypertension which was more prevalent in those with acute coronary syndromes $(79.4 \% ; 49.2 \% ; \mathrm{p}=0.004)$.

\section{DISCUSSION}

Our findings demonstrate that type 2 diabetics comprise $3 \%$ of all medical A\&E attendees, over a six month period, at this tertiary referral facility that also serves as a primary care emergency facility for the majority of the population of the city of Nairobi and its environs, with an estimated population of 3-4 million inhabitants. This is a significant proportion and is consistent with records at this facility and we believe the study reflects the rising prevalenceof type 2 diabetes in our urban and peri-urban populations, for which no comprehensive data exists. By design we opted for the age entry criteria of 30 years to predominantly capture a sample of type 2 diabetes, which we have reason to believe was indeed the case.

A quarter of these diabetic patients demonstrated study designated ischaemic changes in their resting EGG; with the majority showing non-specificST-T wave changes. A minority showed ECG evidence of on-going ischaemia, withSTelevation and presumablynew LBBB pattern; and atrial fibrillation. This high frequency of ischaemic ECG changes is compatible with the high cardiovascular risk profile of the study sample, as demonstrated by their demographics, diabetic vascular age and high prevalence of multiple cardiovascular risk factors. We selected non-specific ischaemic ECG changes as a marker for high CAD risk patient group.

Asimilar high risk profile has been demonstrated in recent studies on type 2 diabetics attending the institution's out-patient diabetic clinics (19). Despite being at high CHD risk, inclusive of significant dyslipidaemia, and the practice guideline recommendations of intensive risk reduction treatment for such patients, the use of statins and mandatory anti-platelet therapy was abysmal. The reasons for this are probably multi-factorial and warrant further study and urgent intervention, in particular regard to the inexpensive and readily accessible acetylsalicylic acid (ASA) therapy.

Consequently a third of these patients were classified as having an ongoing acute coronary syndrome (AGS), on the basis of clinical profile and elevated Troponin assay. This proportion contrast sharply with departmental records, reviewed and compiled as part of the planning stage of this study, which suggested a 7.3\% fourmonth period prevalence(July-October 2006) of AMI in type 2 diabetic attending the A\&E. Despite acknowledging the potential incompleteness of routine data, this discrepancy in prevalence suggests that up to two thirds of such patients are being miss-classified as non-ACS at A\&E presentation evaluation, leading to potential delayed life saving interventions and inappropriate triage; all of which does not portend well 
for favorable outcomes. An important observation is the late and atypical mode of presentation of our ACS in diabetics. Possible reasons for this include the DM associated sensory neuropathy, failure by patients and primaryhealth care workers torecognise the implication of symptoms and inaccessibility to health care; these factors can be potentially overcome by comprehensive health education.

Our data is limited by the potential for type 2 diabetes misclassification bias and tertiary facility referral bias. The performance of C-peptide and Illet cell antibodies studies were unavailable to minimise the latter however, the age range of our sample of 40-87 years and the mean duration of diabetes of 11 years, we believe make the contribution of this bias as nominal. None of ourstudy cases werehospital transfershowever the poor haemodynamic profile of cases should be seen in the light of this inherent referral bias.

In conclusion we have demonstrated that ACS are not infrequent among type 2 diabetics presenting to our A\&E department and that we need to increase vigilance among our staff by imploring a high index of suspicion, given the high risk profile and atypical and varied presentation profile of our patients. We recommend that all diabetics attending our $A \& E$ should undergo a screening ECG evaluation as part of their workup along with a myocardial cell death marker depending on the clinical profile.

\section{ACKNOWLEDGEMENTS}

To J. Anzetse and B. Muia of Department of Internal Medicine, University of Nairobi, Mr. Waithaka and Mr. Njagi of Kenyatta National Hospital laboratory for the analysing the blood samples; Mr. H. Oyugi for data analysis and Director KNH for the opportunity to conduct and publish our work.

\section{REFERENCES}

1. Wild, S., Roglic, G., Green, A., et al. Global prevalence of diabetes: estimates for 2000 and projections for 2030. Diabetes Care. 2004; 27: 1047-1053.

2. Osei, K. Global epidemic of type 2 diabetes: implications for developing countries. Ethn. Dis. 2003; 13: S102-S106.

3. Reddy, K.S. Cardiovascular disease in non - Western countries. N. Engl. J. Med. 2004; 350: 2438 -2440.

4. Yusuf, S., Hawken, S., Ounpuu, S., et al. Effect of potentially modifiable risk factors associated with myocardial infarction in 52 countries The INTERHEART Study: case control study. Lancet. 2004; 364: 937 - 952.

5. Kannel, W.B. and McGee, D.L. Diabetes and cardiovascular risk factors: the Framingham Study: Circulation. 1979; 59: 8-13.

6. De Backer, G., Ambrosioni, E., Borch-Johnsen, K., et al. European guidelines on cardiovascular disease prevention in clinical practice: third joint task force of European and other societies on cardiovascular disease prevention in clinical practice (constituted by representatives ofeight societies and by invited experts): Eur. J. Cardiovasc. Prev. Rehabil. 2003; 10: S1 - S10.

7. Okrainec, K., Banerjee, D.K. and Eisenberg, M.J. Coronary artery disease in the developing world. Am. Heart. J. 2004; 148: 7-15.

8. Steyn, K., Sliwa, K., Hawken, S., et al. Risk factors associated with myocardial infarction in Africa: The INTERHEART Africa study. Circulation. 2005; 112: 3536 - 3540.

9. Smith, S.C., Greenland, P. and Grundy, S.M. Prevention Conference V: Beyond secondary prevention: Identifying the high risk patient for primary prevention: Executive Summary Circulation 2000; 101: 111-116.

10. Koskinen, P., Manttari, M., et al Coronary heart disease incidence in NIDDM patients in the Helsinki Heart Study. Diabetes Care. 1992; 15: 820-825.

11. Haffner, S.M., Lehto, S., Ronnemaa, T., et al. Mortality from coronary heart disease in subjects with type 2 diabetes and in nondiabetic subjects with and without prior myocardial infarction. N. Engl.J. Med. 1998;339: 229-234.

12. Lee, C.D., Folsom, A.R., Pankow, J.S. and Brancati, F.C. Atherosclerosis Risk in Communities (ARIC) Study investigators: Cardiovascular events in diabetic and nondiabetic adults with or without history of myocardial infarction. Circulation. 2004; 109: 855-860.

13. Alpert, J.S., Thygesen, K., Antman, E. and Bassand, J.P. Myocardial infarction redefined - a consensus document of thejointEuropeanSociety of Cardiology/ American College of Cardiology Committee for the redefinition of myocardial infarction. J. Am. Coll. Cardio. 2000; 36: 959 - 969.

14. Scheuner, M.T., Whitworth, W.C., McGruder, H., et al. Familila risk assessment for early-onset coronary heart disease. Genet. Med. 2006; 8: 525-531.

15. Wingard, D.L. and Barrett-Connor, E. Heart disease and diabetes. In: Harris M, ed., Diabetes in America, 2nd ed. National Institute of Health, National Institute of Diabetes and Digestive and Kidney Diseases. NIH Publication. 1995; No. 95 - 1468: 429 - 445.

16. Grundy, S.M., Brewer, H.B. Jr, Cleeman, J.I., et al. American Heart Association; National Heart, Lung, and Blood Institute. The Third Report of the Expert Panel on Detection, Evaluation and Treatment of High Blood Cholesterol in Adults (Adult Treatment Panel III or ATP III) guidelines for the management of serum lipids for high risk patients including those with a coronary artery disease equivalent. Circulation. 2004; 109: 433 - 438.

17. NationalHeart,Lung and Blood Institute. ThePractical Guide: Identification, Evaluation and Treatment of Overweight and Obesity in Adults.US Department of Health and Human Services, Public Health Service, National Institutes of Health, National Heart, Lung and Blood Institute, Bethseda, MD, October 2000.

18. World Health Organisation. Definition, diagnosis and classification of diabetes mellitus and its complications. Report of a WHO Consultation. Part 1: Diagnosis and Classification of Diabetes Mellitus. Geneva: World Health Organisation, 1999.

19. Otieno, C.F., Vaghela, V., Mwendwa, F.W., et al. Cardiovascular risk factors in patients with type 2 diabetes mellitus at the outpatient diabetic clinic of Kenyatta National Hospital, Nairobi. East. Afr. Med. J. 2005; S184 - S189. 